\title{
HISTORIA DE LA CARRERA DE MEDICINA EN LA UNIVERSIDAD NACIONAL AUTONOMA DE HONDURAS EN EL VALLE DE SULA
}

\section{History of Medical Career at Sula Valley National Autonomous University of Honduras}

\author{
José Raúl Arita Chávez*
}

\section{INTRODUCCIÓN}

La Carrera de Medicina en el Centro Universitario Regional del Norte (hoy UNAH-VS), nace para dar respuesta principalmente a las necesidades de salud y educación de la zona nor-occidental del país. La creación de esta carrera ha generado gran interés y expectativas en la sociedad de las regiones norte y occidente del país, ya que para lograr su gestación se contó con el apoyo y participación de diferentes sectores como: La Fundación Sampedrana de la Escuela de Medicina (FUSEMED), las direcciones de los hospitales Dr. Mario Catarino Rivas (HNMCR), Hospital Leonardo Martínez Valenzuela (HLMV) y Organización de Estudiantes de Medicina matriculados en el CURN. La iniciativa de estos sectores fue finalmente considerada por la Comisión de Transición de la UNAH, la Rectoría, la Junta Interventora e Investigadora de la Facultad de Ciencias Médicas y la Decanatura de la misma. Así mismo, el Colegio Médico de Honduras mostró un creciente interés en la consolidación de la Carrera de Medicina. Igualmente otras fuerzas vivas de San Pedro Sula, específicamente; la Cámara de Comercio e Industrias de Cortés con su participación desarrollaron un papel de mediadora en las fases finales del proceso de creación de la carrera por las autoridades universitarias. (1)

\footnotetext{
* Profesor / Jefe de la Escuela Universitaria de las Ciencias de la Salud de la UNAH-VS, Médico y Cirujano, Master en Educación Superior. Dirigir correspondencia a: jaritac@unah.edu.hn Recibido: 8 de Julio 2,015 Aprobado 21 de Julio 2,015
}

\section{RESEÑA HISTORICA DE LA CARRERA DE MEDICINA EN UNAH-VS}

La Carrera de Medicina solo existía en la Ciudad Universitaria de la UNAH como institución pública; bajo la estructura de La Facultad de Ciencias Médicas (FCM) que fue fundada el 14 de febrero de 1882 durante el gobierno del Dr. Marco Aurelio Soto, siendo su Ministro de instrucción pública el Dr. Ramón Rosa, el Rector de la Universidad el Dr. Adolfo Zúñiga y el Decano de la Facultad de Medicina el Dr. Carlos Ernesto Bernhan. (2)

Ante la necesidad de la población de la zona Nor-Occidental de contar con la Carrera de Medicina, se hace solicitud al Consejo Universitario quien remite dicha petición al Decanato de Facultad de Ciencias Médicas, y este integra una comisión para que realice el análisis correspondiente.

La comisión realiza un estudio y presenta el informe con fecha 1 de diciembre del 2003, el cual contiene los siguientes puntos:

a. Que en reunión sostenida con el Lic. Carlos Gallegos Director del CURN (Hoy UNAH/VS) y el Dr. Pastor Laínez Coordinador del Internado Rotatorio en el HNMCR argumentan que aproximadamente el $50 \%$ de los estudiantes que cursan la Carrera de Medicina en Tegucigalpa proceden de la zona nor-occidental y que cuentan con el apoyo de los padres de familia de dichos jóvenes. 
b. La comisión se reúne con el Lic. Luis Barahona, Director Suplente de la Dirección de Educación Superior, quien expuso las condiciones requeridas para la creación de una nueva carrera, por lo que aconseja hablar de extensión de la Carrera de Medicina, y así la Facultad de Ciencias Médicas tendría la facultad de supervisar, evaluar y emitir opiniones sobre el curso que tome el estudio de Medicina en San Pedro Sula.

c. Posteriormente la comisión se traslada a San Pedro Sula, donde fue recibida por autoridades del CURN, del HMCR, HLMV y Asociación de Padres de Familia de futuros estudiantes de medicina; encontrando condiciones de aulas, laboratorios y recurso humano para poder iniciar los estudios de medicina, además de la anuencia por autoridades y personal médico de dichos hospitales y de la asociación de padres de familia quienes ofrecen su concurso de tipo económico para sufragar los gastos que implica la contratación de docentes.

El apoyo ofrecido por la Dirección del Hospital Dr. Mario Catarino Rivas se encuentra en el oficio No. 194-03 D/HNMCR de fecha 09 de septiembre de 2003 el cual fue enviado al Dr. Pastor Laínez, Coordinador del Proyecto de la Carrera de Medicina en el CURN. También existe nota dirigida al Dr. Pastor Laínez, la que es enviada por el Dr. José Samara, Director del Hospital Leonardo Martínez Valenzuela, en la cual manifiesta la anuencia de esa dirección a colaborar con la Carrera de Medicina. En base a los puntos anteriores la comisión concluye que se está de acuerdo con la extensión de la Carrera de Medicina en San Pedro Sula, misma que debe iniciarse, de preferencia, de manera progresiva, es decir comenzando por el área de ciencias morfológicas y para la administración del plan de estudios de la carrera de medicina en el CURN se tendrá que nombrar un coordinador general de la carrera.

Posteriormente la comisión proyectista presentó a la Dirección de Docencia el documento justificativo y mediante dictamen emitido a los diez días del mes de diciembre, recomienda al Consejo Universitario: Aprobar la ampliación de la Carrera de Medicina de la FCM para ser realizada en el CURN en coordinación con el HMCR, HLMV y la Región Sanitaria No. 3, por considerar que se cuenta con el espacio físico, recursos humanos y económicos para iniciar su funcionamiento, que académicamente la carrera dependa de la FCM en Tegucigalpa y que se aplique en su totalidad el Plan de Estudios vigente de la Carrera de Medicina que se encuentra debidamente registrado, foliado y sellado en la Secretaría General.

Ante el dictamen de la Dirección de Docencia, el Honorable Consejo Universitario en sesión ordinaria celebrada el día 11 y 18 de diciembre de 2003 adopta el acuerdo No. 126-2003-CUO que dice "Aprobar la Ampliación de la Carrera de Medicina de la Facultad de Ciencias Médicas para ser realizada en el Centro Universitario Regional del Norte CURN, en coordinación con el Hospital Nacional Dr. Mario Catarino Rivas, Hospital Dr. Leonardo Martínez Valenzuela y la Región Sanitaria No. 3".

El Honorable Consejo Universitario, en su sesión ordinaria, celebrada el día jueves 27 de mayo de 2004, mediante acuerdo No. 
050-2004-CUO y que consta en el acta No. 779, modifica el acuerdo No. 019-2004-CUO del acta No. 776 , en lo relativo a la integración de la comisión que realiza un estudio en relación a la apertura de la Carrera de Medicina en el CURN, la cual deberá estar integrada por el Dr. Octavio Rubén Sánchez, docente de la FCM, quien la coordinará, Dr. Gustavo Vallejo, docente de la FCM, Dr. Renato Valenzuela Castillo, docente de la FCM, Abogado Rigoberto Espinal Irías, docente de la Facultad de Ciencias Jurídicas y Sociales, Dr. Jorge Haddad Quiñónez, exdecano de la FCM, como asesor y los estudiantes: $\mathrm{Br}$. Lenin Castañeda, Br. Henry Ponce, Br. Osiris Jesua Peña y el Br. Rodrigo Rodríguez.

Esta comisión realizó un taller de 3 días en el Lago de Yojoa en el cual se socializó e informó sobre el proyecto de ampliación de la Carrera de Medicina desde Tegucigalpa al CURN de San Pedro Sula. Además se identificaron las fortalezas y debilidades que tenía la FCM en Tegucigalpa para formar médicos y las fortalezas y debilidades que tenía el CURN de San Pedro Sula para apoyar la ampliación de la Carrera de Medicina. Se definieron los elementos más relevantes para la elaboración de un Plan Estratégico de la ampliación de la Carrera de Medicina al CURN, se elaboró un cronograma para el desarrollo del Plan Estratégico, informándose sobre las tendencias actuales en el proceso enseñanza-aprendizaje de la Carrera de Medicina, y definiéndose la estructura académico-administrativa que debería dirigir el proceso de ampliación de la Carrera de Medicina desde Tegucigalpa a San Pedro Sula así como los principales componentes que debería tener un Plan de Profesionalización y Capacitación de los docentes de la FCM de Tegucigalpa y del CURN.

Para continuar con las gestiones sobre la ampliación de la Carrera de Medicina en el CURN, la Fundación Sampedrana de la Escuela de Medicina obtuvo su Personería Jurídica y aprobación de sus estatutos mediante resolución No. 427-2005 del despacho de Gobernación y Justicia en fecha 26 de abril de 2005. El objetivo de la Fundación fue apoyar financieramente la construcción de la infraestructura requerida y su equipamiento para el cumplimiento del programa educativo de la Carrera de Medicina. Lográndose lo anterior se elaboró el proyecto de convenio entre la Universidad Nacional Autónoma de Honduras (UNAH) y la Fundación Sampedrana de la Escuela de Medicina (FUSEMED), para el funcionamiento de la ampliación de la Carrera de Medicina en el Centro Universitario Regional del Norte, en la Cuidad de San Pedro Sula; documento que fue presentado al Lic. Pablo Ulises Gómez Velásquez, Secretario Ejecutivo de Administración y Finanzas, quien a su vez lo remite mediante oficio No. $691 \mathrm{del}$ 18 de julio de 2005 al Abogado General de la UNAH, Señor Francisco Galdámez Monge, para que emita dictamen sobre el proyecto de convenio entre la UNAH y FUSEMED.

El abogado Galdámez Monge en respuesta a solicitud de dictamen, emite el oficio No. 188/05 del 28 de julio de 2005, en el cual hace algunas observaciones y propone que FUSEMED no tenga injerencia académica y administrativa, relativa al funcionamiento de la Carrera de Medicina del CURN.

Por lo que recomienda utilizar como guía el 
oficio A. L. No.181-05 del 25 de julio de 2005, para que no haya atraso en que FUSEMED haga efectivo su cooperación a la Carrera de Medicina del CURN.

La Carrera de Medicina en el CURN, inició y culminó las clases del área de ciencias morfológicas en el segundo semestre del año 2005 , sin embargo por irregularidades cometidas en el proceso de ampliación de la carrera, como incumplimiento en algunas de las disposiciones de la Ley Orgánica, Ley de Educación Superior, Normas Académicas de la Educación Superior, Ley Orgánica del Colegio Médico, Estatuto del Docente y Reglamento de la Facultad de Ciencias Médicas, como por la existencia de limitantes en la infraestructura física de aulas y laboratorios en el CURN, la Comisión de Transición de la UNAH, en su acuerdo No. 38 y por unanimidad de votos acordó en el 1 de diciembre de 2005 suspender por un año los efectos del acuerdo No. CUO 126-2003 emitido por el Consejo Universitario y contenido en el punto No. 8 del acta No. 773 del 11 y 18 de diciembre de 2004, que versa sobre la autorización para el funcionamiento de la extensión de la Carrera de Medicina en el CURN. También se acuerda que la Junta Interventora e Investigadora de la FCM elaboré un plan orientado a que se cumpla con todos los requisitos académicos, legales, laborales y financieros necesarios para garantizar la viabilidad técnica, financiera y llenar los criterios de sostenibilidad de su desarrollo, con la máxima calidad posible, de la Carrera de Medicina en el CURN. Asimismo la Junta Interventora e Investigadora, en el ámbito de sus atribuciones es la responsable de velar $y$ coordinar la correcta ejecución de las medidas contempladas en el presente acuerdo
El 18 de enero del 2006 el presidente de la Comisión de transición, la Coordinadora de la Junta Interventora e Investigadora de la Facultad de Ciencias Médicas por una parte y el Vocal Primero de la Junta Directiva de la FUSEMED firmaron un acta única en la que se establece que se solicita de mutuo acuerdo la intervención de la Cámara de Comercio e Industrias de Cortés, a efecto que esta actúe como Conciliadora en el proceso que se desarrollará para definir e implementar las acciones necesarias, oportunas y pertinentes enfocadas a la apertura normada y organizada de la Carrera.

En fecha 3 de febrero de 2006 se firmó un acta de compromiso por el Rector de la UNAH, el Presidente de la Comisión de Transición, la Coordinadora de la Facultad de ciencias Médicas, la Presidente de la junta directiva de FUSEMED, los representantes del Comisionado de Alto Nivel del Consejo Universitario de la UNAH y el Presidente de Asociación de Estudiantes de Medicina del CURN, en la cual los firmantes se comprometieron a trabajar armónicamente para que se reinicie la Carrera de Medicina en el CURN y evaluar periódicamente el proceso, para que la carrera comience en agosto del 2006, con docentes empleados directamente de la UNAH, de tal manera que se realizó el concurso para los docentes de ciencias morfológicas en la FCM a inicios del 2006, siendo contratados de manera permanente a partir del 1 de Abril para el desarrollo de una jornada de inducción y capacitación disciplinar y pedagógica durante cuatro meses y luego en Agosto de este mismo año inicia la carrera con las clases de ciencias morfológicas. ${ }^{(3)}$

Con la aprobación de inicio de clases de las 
asignaturas de Ciencias Morfológicas para agosto del año 2006, se nombró el Coordinador de la Carrera y un Equipo de Docentes comprometido con el crecimiento, desarrollo y calidad de la carrera. (Ver Figura No. 1.)

Al interior del CURN se contó con el apoyo de sus autoridades y de las unidades academicas de docencia, investigación y extensión. Vale la pena señalar el interés que tenían otras carreras del área de la salud como Enfermería y Odontología, en realizar esfuerzos conjuntos para desarrollar proyectos académicos que contribuyeran a mejorar las condiciones de vida y salud de la comunidad universitaria y de aquellas comunidades de San Pedro Sula más desprotegidas. Este interés permitió que en el CURN se unieran esfuerzos de los talentos humanos, en la infraestructura física, equipo y materiales, para lograr la creación de la Escuela de Ciencias de la Salud, elemento que permitiría formar equipos de salud competentes para transformar la problemática de salud de la sociedad. El agradecimiento a ellos fue expresado por Dr. Francisco J. Herrera Alvarado, Coordinador de la Carrera de Medicina-CURN quien textualmente expreso:

Agradecemos el apoyo brindado por todos para el inicio de la Carrera de Medicina, y esperamos que nuevos sectores importantes de la sociedad comprometidos con la calidad de la atención integral en salud, e interesados en lograr que en esta ciudad, considerada de manera indiscutible un importantísimo polo de desarrollo de la región centroamericana; se incorporen en forma activa y participativa. La meta es y será siempre la formación de profesionales íntegros, científicamente hábiles y socialmente sensibles. ${ }^{(1)}$

El plan de estudios con el que inició la carrera y aún está vigente es el del año 2000 implementado a partir del año 2001 y que surge de la readecuación del Plan 95 original (Readecuación 2000) que consta de 68 asignaturas, de las cuales 4 son generales pétreas, 13 son generales sustantivas, 7 son de salud pública, 16 son de ciencias básicas de la salud y 28 son del área de medicina clínica integral, con un peso de 409 Unidades Valorativas (U.V.) distribuidas así: No. U.V. teóricas 292(Horas Teóricas); 5,256 = 45.4\%, No. U.V. Prácticas 117 (Horas Práctica); $6,318=54.6 \%$; haciendo un total de horas que asciende a 11,574.

La Duración de la Carrera es de 8 años distribuidos en 8 períodos académicos de 18 semanas cada uno del I al IV año de la Carrera, 6 pasantías de 7 semanas cada una para $V$ año, 8 pasantías de 5 semanas cada una para $\mathrm{VI}$ año y 4 rotaciones de 13 semanas cada una para VII año y finalmente 1 año de Servicio Social.

Así mismo en el año 2012 el Consejo Universitario resuelve aprobar el reglamento del internado rotatorio en el ACTA NÚMERO CU-O-006-06-2012 y ACUERDO No. CU-O-025-02-2012.

Además el Servicio Social se rige bajo el Reglamento de Servicio Médico Social Obligatorio aprobado por la Junta Directiva de la Facultad de Ciencias Médicas el 23 de Agosto de 1969, reformado y aprobado de nuevo el 17 de Marzo de 1992, y finalmente se le realizaron reformas, reconfirmadas y 
aprobadas el 11 de Octubre del 2000 con vigencia actual. ${ }^{(4)}$

La Acreditación de la Carrera es de Título de Doctor en Medicina y Cirugía, ampliamente discutido por el Consejo Universitario durante el 2014 antes de la aprobación de las normas académicas (aprobadas bajo el Acuerdo No. CU-E-107-09-2014), para finalmente establecer en el Artículo 131 donde se establecen los grados académicos en el nivel de educación superior como: Doctor en Medicina y Cirugía. ${ }^{(5)}$ En abril del 2006 se inició con una planta docente conformada por 7 profesores distribuidos así, como Coordinador de la Carrera de Medicina Dr. Francisco J. Herrera A; Docente del Área de Salud Pública Dra. Elba R. Morán G; Docentes del Área de Ciencias Morfológicas Dra. Aída R. Merlo, Dra. Brenda L. Solórzano, Dr. José Raúl Arita, Dr. F. Javier Sánchez B y Dra. Jessica P. Sánchez. (Ver Figura No. 1) $)^{(1)}$ Dicha planta de docentes fue creciendo paulatinamente de acuerdo a las necesidades en cada periodo académico a tal grado que actualmente la carrera cuenta con el servicio de 75 profesores. ${ }^{(6)}$
Figura No. 1: Equipo docente que inició en la Carrera de Medicina y autoridades universitarias.

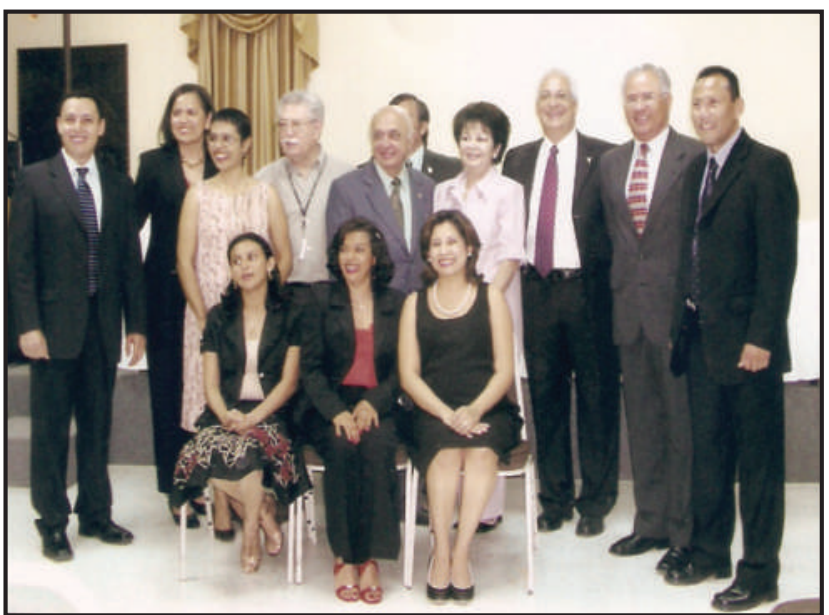

Se encuentran: Miembros de la Comisión de Transición, Representantes de la Facultad de Ciencias Médicas y Planta Docente inicial.

En Agosto del 2006 con el inicio de las ciencias morfológicas la matricula se realizó con un cupo limitado de únicamente 40 estudiantes de excelencia académica, quienes fueron aperturando cada periodo académico, para el 2011 la carrera ha completado el séptimo año que equivale al internado rotatorio, contando con Médicos en Servicio Social para el año 2012, hasta culminar, juramentarse en ceremonia solemne y graduarse en el 2013 un total de 29 médicos, (Ver Figura No. 2) ${ }^{(7)}$ así han continuado finalizando haciendo un total para el 2015 de 102 médicos graduados. ${ }^{(8)}$

Figura No. 2: Primera graduación de Medicina EUCS, UNAH-VS.

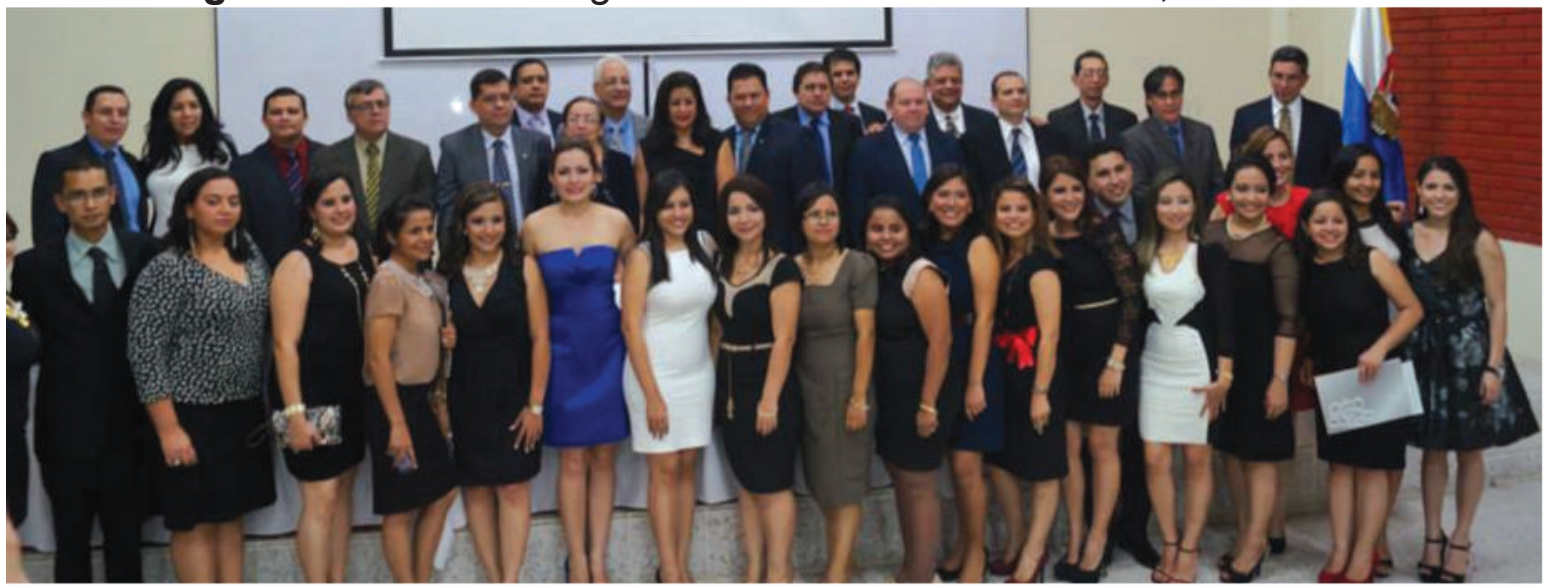

Se encuentran: graduandos acompañados por docentes y autoridades que asistieron al evento, el cual se realizó el viernes 06 de septiembre de 2013 
Por resolución de la Comisión de Transición de la UNAH, a partir del 1 de febrero de 2007, el CURN se convierte en la Universidad Nacional Autónoma de Honduras en el Valle de Sula, (UNAH-VS). ${ }^{(9)}$ La Carrera de Medicina pasa a formar parte de la estructura organizativa de la Escuela Universitaria de las Ciencias de la Salud (EUCS) de la UNAH-VS, creada en febrero del 2008 bajo el "acuerdo N 348-A-2008" de la Comisión de Transición de la UNAH. ${ }^{(10)}$

En la Carrera de Medicina, hasta antes del 2007 los estudiantes eran admitidos como en las demás carreras de la UNAH, únicamente expresando que deseaban estudiar determinada profesión, a partir de este año el ingreso se encuentra sujeto al Sistema de Admisión de la UNAH con la aplicación de la Prueba de Aptitud Académica (PAA) obteniendo un mínimo de 1000 puntos, luego este puntaje fue modificado a 1100 puntos, más la Prueba de Conocimientos en Ciencias Naturales y de la Salud (PCCNS) para el año 2012, Calculando el resultado final con la siguiente fórmula: Puntaje para selección $=$ Resultado de la ((PAA $\times 100 / 1600)+$ (resultado de la prueba de conocimiento $\times 100 / 800)$ ) / 2 .

En cumplimiento al acuerdo número CU-O-121-12-2011 del Consejo Universitario emitido el día 13 de diciembre del 2011 quien aprobó la implementación de cupo en la Carrera de Medicina y fue ratificado el 22 de mayo 2012, se definió para la Carrera de Medicina a partir del tercer período académico del $2012 \mathrm{el}$ cupo máximo anual de 100 estudiantes en UNAH-VS cincuenta (50) estudiantes en el primer periodo académico y cincuenta (50) estudiantes en el tercer periodo académico.

Esta normativa se aplicó a partir del proceso de la PAA para ingreso y matrícula en el tercer período del 2012, de tal manera que el establecimiento de la misma ha permitido la mejora continua de la calidad y ha brindado la oportunidad de disminuir la masificación de la población estudiantil acumulada en los últimos años. ${ }^{(11)}$

El comportamiento de la matrícula desde el inicio de la carrera en el 2006, tomando como referencia únicamente la matrícula del primer período académico de cada año, mostró un ascenso continuo hasta alcanzar su mayor nivel de 1534 estudiantes en el 2012, luego inicia el descenso en el 2013 hasta la actualidad con 868 estudiantes matriculados en el 2015 (Ver Gráfica No 1.), lo que responde a los criterios de admisión implementados. ${ }^{(12)}$

Gráfica No 1: Matrícula primer período de cada año. (2006-2015)

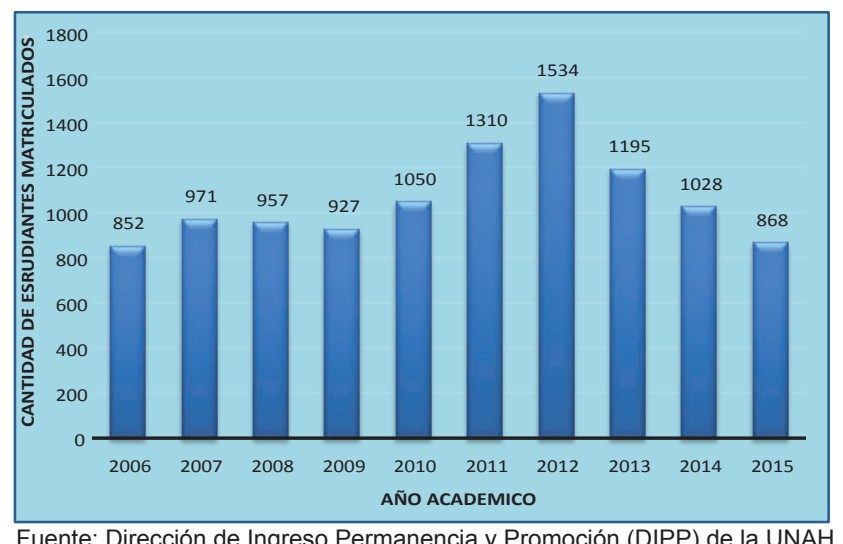

El proceso de Autoevaluación de la Carrera de Medicina de la UNAH/VS, se desarrolló de manera intermitente a partir de Diciembre de 2007 hasta culminar en julio del 2012, trabajo desarrollado por las tres subcomisiones conformadas en este período de tiempo, Primera Subcomisión: Dr. Francisco J. Herrera, Dr. Fidel Bulnes, Dra. Rossany E. Escalante, Lic. Lorena Córdova, Dr. Marco A. Fajardo; Segunda Subcomisión: Dr. Francisco J. Herrera, Dr. Fidel Bulnes, Dra. Ro- 
ssany E. Escalante; Tercera Subcomisión: Dr. Francisco J. Herrera, Dra. Silvia Piura, Dr. Fidel Bulnes, Dr. Marvin Rodríguez; avanzando actualmente en el plan de mejora respectivo. ${ }^{(3)}$

En la actualidad se encuentra en proceso de rediseño curricular en el que las reformas curriculares responden a los cambios sociales del patrón epidemiológico y la evolución de la educación médica a nivel mundial, vinculadas a la mejoría de las condiciones de salud de la población.

La comisión de desarrollo curricular actual está conformada por: Dr. Francisco Herrera
A., Dr. Pastor Laínez, Dr. José Raúl Arita, Dr. Roberto Pineda Leiva y Dr. Marvin Rodríguez.

A partir de Abril del 2014 la Coordinación de la Carrera está a cargo del Dr. José Pastor Laínez Macis.

Respondiendo a la Internacionalización, durante el segundo semestre del 2014 a través de la Vicerrectoría Académica se realizó la movilidad académica bajo el enfoque de AMIDILA (Academic Mobility end Inclusive Development in Latin America) con el becario Luca Giudice, de la Universidad Di Padova Italy quien cursó clases de $V$ año de la Carrera de Medicina. ${ }^{(7)}$

\section{BIBLIOGRAFÍA}

1. Boletín Informativo de la Carrera de Medicina del CURN Médicos del Siglo XXI, agosto - diciembre 2006.

2. Documento Base de Rediseño Curricular Facultad de Ciencias Médicas, Tegucigalpa, Honduras. Junio 2013. 17

3. Informe del Proceso de Autoevaluación de la Carrera de Medicina San Pedro Sula, Cortés, Honduras, 31 de julio del 2012.

4. Plan de Estudio del Pre-grado de Medicina Código-2000 Readecuación-2000, estructura del plan de estudios, página 77, Tegucigalpa Honduras, 2013.

5. Normas Académicas, UNAH, Noviembre 2014

6. Estadísticas o archivo de coordinación de Carrera Medicina, Universidad Nacional Autónoma de Honduras en el Valle de Sula, UNAH-VS, Junio, 2015.
7. Estadísticas de la secretaría de la Universidad Nacional Autónoma de Honduras en el Valle de Sula, UNAH-VS, Junio 2015.

8. Base de datos y estadísticas de la Escuela Universitaria de las Ciencias de la Salud, EUCS de la Universidad Nacional Autónoma de Honduras en el Valle de Sula, UNAH-VS, junio 2015.

9. Modelo Teórico de la UNAH-VS, Comisión de Apoyo a la Reforma Universitaria, San Pedro Sula, Honduras, marzo 2008.

10. Proyecto de creación de la Escuela Universitaria de Ciencias de la Salud (EUCS) de la UNAH-VS, San Pedro Sula, Honduras, 2008.

11. Boletín Informativo, UNAH, Facultad de Ciencias Médicas, enero 2013.

12. Estadísticas de la Dirección de Ingreso Permanencia y Promoción, DIIP de la Universidad Nacional Autónoma de Honduras, junio 2015. 